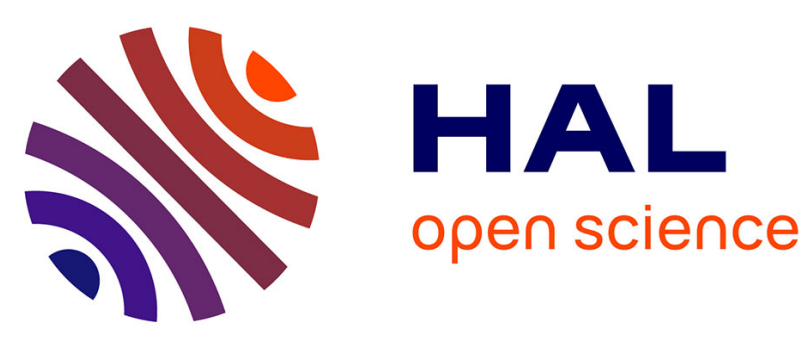

\title{
Anderson Localization of Expanding Bose-Einstein Condensates in Random Potentials
}

Laurent Sanchez-Palencia, David Clément, Pierre Lugan, Philippe Bouyer, Georgy V. Shlyapnikov, Alain Aspect

\section{- To cite this version:}

Laurent Sanchez-Palencia, David Clément, Pierre Lugan, Philippe Bouyer, Georgy V. Shlyapnikov, et al.. Anderson Localization of Expanding Bose-Einstein Condensates in Random Potentials. Physical Review Letters, 2007, 98, pp.210401. 10.1103/PhysRevLett.98.210401 . hal-00122278v4

\section{HAL Id: hal-00122278 \\ https://hal.science/hal-00122278v4}

Submitted on 23 May 2007

HAL is a multi-disciplinary open access archive for the deposit and dissemination of scientific research documents, whether they are published or not. The documents may come from teaching and research institutions in France or abroad, or from public or private research centers.
L'archive ouverte pluridisciplinaire HAL, est destinée au dépôt et à la diffusion de documents scientifiques de niveau recherche, publiés ou non, émanant des établissements d'enseignement et de recherche français ou étrangers, des laboratoires publics ou privés. 


\title{
Anderson Localization of Expanding Bose-Einstein Condensates in Random Potentials
}

\author{
L. Sanchez-Palencia, ${ }^{1}$ D. Clément, ${ }^{1}$ P. Lugan, ${ }^{1}$ P. Bouyer, ${ }^{1}$ G.V. Shlyapnikov, ${ }^{2,3}$ and A. Aspect ${ }^{1}$ \\ ${ }^{1}$ Laboratoire Charles Fabry de l'Institut d'Optique, CNRS and Univ. Paris-Sud, \\ Campus Polytechnique, RD 128, F-91127 Palaiseau cedex, France \\ ${ }^{2}$ Laboratoire de Physique Théorique et Modèles Statistiques, Univ. Paris-Sud, F-91405 Orsay cedex, France \\ ${ }^{3}$ Van der Waals-Zeeman Institute, Univ. Amsterdam, Valckenierstraat 65/67, 1018 XE Amsterdam, The Netherlands
}

(Dated: 23rd May 2007)

\begin{abstract}
We show that the expansion of an initially confined interacting 1D Bose-Einstein condensate can exhibit Anderson localization in a weak random potential with correlation length $\sigma_{\mathrm{R}}$. For speckle potentials the Fourier transform of the correlation function vanishes for momenta $k>2 / \sigma_{\mathrm{R}}$ so that the Lyapunov exponent vanishes in the Born approximation for $k>1 / \sigma_{\mathrm{R}}$. Then, for the initial healing length of the condensate $\xi_{\text {in }}>\sigma_{\mathrm{R}}$ the localization is exponential, and for $\xi_{\text {in }}<\sigma_{\mathrm{R}}$ it changes to algebraic.
\end{abstract}

PACS numbers: 05.30.Jp,03.75.Kk,03.75.Nt,05.60.Gg

Disorder in quantum systems can have dramatic effects, such as strong Anderson localization (AL) of non-interacting particles in random media [1]. The main paradigm of AL is that the suppression of transport is due to a destructive interference of particles (waves) which multiply scatter from the modulations of a random potential. AL is thus expected to occur when interferences play a central role in the multiple scattering process [2]. In three dimensions, this requires the particle wavelength to be larger than the scattering mean free path, $l$, as pointed out by Ioffe and Regel [3]. One then finds a mobility edge at momentum $k_{\mathrm{m}}=1 / l$, below which AL can appear. In one and two dimensions, all single-particle quantum states are predicted to be localized [月, 5, 6], although for certain types of disorder one has an effective mobility edge in the Born approximation (see Ref. [7] and below). A crossover to the regime of AL has been observed in low dimensional conductors [8, 9], and recently, evidences of AL have been obtained for light waves in bulk powders [10] and in 2D disordered photonic lattices [11]. The subtle question is whether and how the interaction between particles can cause delocalization and transport, and there is a long-standing discussion of this issue for the case of electrons in solids [12].

Ultracold atomic gases can shed new light on these problems owing to an unprecedented control of interactions, a perfect isolation from a thermal bath, and the possibilities of designing controlled random [13, 14, 15, 16, 17] or quasirandom [18] potentials. Of particular interest are the studies of localization in Bose gases [19, 20] and the interplay between interactions and disorder in Bose and Fermi gases [21. 22]. Localization of expanding Bose-Einstein condensates (BEC) in random potentials has been reported in Refs. [15, 16, 17]. However, this effect is not related to AL, but rather to the fragmentation of the core of the BEC, and to single reflections from large modulations of the random potential in the tails [15]. Numerical calculations [15, 23, 24] confirm this scenario for parameters relevant to the experiments of Refs. [15, 16, 17].

In this Letter, we show that the expansion of a 1D interacting $\mathrm{BEC}$ can exhibit AL in a random potential without large or wide modulations. Here, in contrast to the situation in
Refs. 115, 16, 17, the BEC is not significantly affected by a single reflection. For this weak disorder regime we have identified the following localization scenario on the basis of numerical calculations and the toy model described below.

At short times, the disorder does not play a significant role, atom-atom interactions drive the expansion of the BEC and determine the long-time momentum distribution, $\mathcal{D}(k)$. According to the scaling theory [25], $\mathcal{D}(k)$ has a highmomentum cut-off at $1 / \xi_{\text {in }}$, where $\xi_{\text {in }}=\hbar / \sqrt{4 m \mu}$ and $\mu$ are the initial healing length and chemical potential of the BEC, and $m$ is the atom mass. When the density is significantly decreased, the expansion is governed by the scattering of almost non-interacting waves from the random potential. Each wave with momentum $k$ undergoes AL on a momentum-dependent length $L(k)$ and the BEC density profile will be determined by the superposition of localized waves. For speckle potentials the Fourier transform of the correlation function vanishes for $k>2 / \sigma_{\mathrm{R}}$, where $\sigma_{\mathrm{R}}$ is the correlation length of the disorder, and the Born approach yields an effective mobility edge at $1 / \sigma_{\mathrm{R}}$. Then, if the high-momentum cut-off is provided by the momentum distribution $\mathcal{D}(k)$ (for $\xi_{\text {in }}>\sigma_{\mathrm{R}}$ ), the BEC is exponentially localized, whereas if the cut-off is provided by the correlation function of the disorder (for $\xi_{\text {in }}<\sigma_{\mathrm{R}}$ ) the localization is algebraic. These findings pave the way to observe AL in experiments similar to those of Refs. [15, 16, 17].

We consider a 1D Bose gas with repulsive short-range interactions, characterized by the 1D coupling constant $g$ and trapped in a harmonic potential $V_{\mathrm{ho}}(z)=m \omega^{2} z^{2} / 2$. The finite size of the trapped sample provides a low-momentum cut-off for the phase fluctuations, and for weak interactions ( $n \gg m g / \hbar^{2}$, where $n$ is the $1 \mathrm{D}$ density), the gas forms a true $\mathrm{BEC}$ at low temperatures [26].

We treat the BEC wave function $\psi(z, t)$ using the GrossPitaevskii equation (GPE). In the presence of a superimposed random potential $V(z)$, this equation reads:

$$
i \hbar \partial_{t} \psi=\left[\frac{-\hbar^{2}}{2 m} \partial_{z}^{2}+V_{\mathrm{ho}}(z)+V(z)+g|\psi|^{2}-\mu\right] \psi,
$$

where $\psi$ is normalized by $\int \mathrm{d} z|\psi|^{2}=N$, with $N$ being the number of atoms. It can be assumed without loss of gener- 
ality that the average of $V(z)$ over the disorder, $\langle V\rangle$, vanishes, while the correlation function $C(z)=\left\langle V\left(z^{\prime}\right) V\left(z^{\prime}+z\right)\right\rangle$ can be written as $C(z)=V_{\mathrm{R}}^{2} c\left(z / \sigma_{\mathrm{R}}\right)$, where the reduced correlation function $c(u)$ has unity height and width. So, $V_{\mathrm{R}}=\sqrt{\left\langle V^{2}\right\rangle}$ is the standard deviation, and $\sigma_{\mathrm{R}}$ is the correlation length of the disorder.

The properties of the correlation function depend on the model of disorder. Although most of our discussion is general, we mainly refer to a 1D speckle random potential [27] similar to the ones used in experiments with cold atoms $113,14,15,16,17$, . It is a random potential with a truncated negative exponential single-point distribution [27]:

$$
\mathcal{P}[V(z)]=\frac{\exp \left[-\left(V(z)+V_{\mathrm{R}}\right) / V_{\mathrm{R}}\right]}{V_{\mathrm{R}}} \Theta\left(\frac{V(z)}{V_{\mathrm{R}}}+1\right),
$$

where $\Theta$ is the Heaviside step function, and with a correlation function which can be controlled almost at will [17]. For a speckle potential produced by diffraction through a 1D square aperture [17, 27], we have

$$
C(z)=V_{\mathrm{R}}^{2} c\left(z / \sigma_{\mathrm{R}}\right) ; \quad c(u)=\sin ^{2}(u) / u^{2} .
$$

Thus the Fourier transform of $C(z)$ has a finite support:

$\widehat{C}(k)=V_{\mathrm{R}}^{2} \sigma_{\mathrm{R}} \widehat{c}\left(k \sigma_{\mathrm{R}}\right) ; \widehat{c}(\kappa)=\sqrt{\pi / 2}(1-\kappa / 2) \Theta(1-\kappa / 2)$,

so that $\widehat{C}(k)=0$ for $k>2 / \sigma_{\mathrm{R}}$. This is actually a general property of speckle potentials, related to the way they are produced using finite-size diffusive plates [27].

We now consider the expansion of the BEC, using the following toy model. Initially, the BEC is assumed to be at equilibrium in the trapping potential $V_{\mathrm{ho}}(z)$ and in the absence of disorder. In the Thomas-Fermi regime (TF) where $\mu \gg \hbar \omega$, the initial BEC density is an inverted parabola, $n(z)=(\mu / g)\left(1-z^{2} / L_{\mathrm{TF}}^{2}\right) \Theta\left(1-|z| / L_{\mathrm{TF}}\right)$, with $L_{\mathrm{TF}}=$ $\sqrt{2 \mu / m \omega^{2}}$ being the TF half-length. The expansion is induced by abruptly switching off the confining trap at time $t=0$, still in the absence of disorder. Assuming that the condition of weak interactions is preserved during the expansion, we work within the framework of the GPE (11). Repulsive atom-atom interactions drive the short-time $(t \lesssim 1 / \omega)$ expansion, while at longer times $(t \gg 1 / \omega)$ the interactions are not important and the expansion becomes free. According to the scaling approach [25], the expanding BEC acquires a dynamical phase and the density profile is rescaled, remaining an inverted parabola:

$$
\psi(z, t)=(\psi[z / b(t), 0] / \sqrt{b(t)}) \exp \left\{\mathrm{i} m z^{2} \dot{b}(t) / 2 \hbar b(t)\right\},
$$

where the scaling parameter $b(t)=1$ for $t=0$, and $b(t) \simeq$ $\sqrt{2} \omega t$ for $t \gg 1 / \omega[15$.

We assume that the random potential is abruptly switched on at a time $t_{0} \gg 1 / \omega$. Since the atom-atom interactions are no longer important, the BEC represents a superposition of almost independent plane waves:

$$
\psi(z, t)=\int \frac{\mathrm{d} k}{\sqrt{2 \pi}} \widehat{\psi}(k, t) \exp (\mathrm{i} k z) .
$$

The momentum distribution $\mathcal{D}(k)$ follows from Eq. (5). For $t \gg 1 / \omega$, it is stationary and has a high-momentum cut-off at the inverse healing length $1 / \xi_{\text {in }}$ :

$$
\mathcal{D}(k)=|\widehat{\psi}(k, t)|^{2} \simeq \frac{3 N \xi_{\text {in }}}{4}\left(1-k^{2} \xi_{\text {in }}^{2}\right) \Theta\left(1-k \xi_{\text {in }}\right),
$$

with the normalization condition $\int_{-\infty}^{+\infty} \mathrm{d} k \mathcal{D}(k)=N$.

According to the Anderson theory [1], $k$-waves will exponentially localize as a result of multiple scattering from the random potential. Thus, components $\exp (\mathrm{i} k z)$ in Eq. (6) will become localized functions $\phi_{k}(z)$. At large distances, $\phi_{k}(z)$ decays exponentially, so that $\ln \left|\phi_{k}(z)\right| \simeq-\gamma(k)|z|$, with $\gamma(k)=1 / L(k)$ the Lyapunov exponent, and $L(k)$ the localization length. The AL of the BEC occurs when the independent $k$-waves have localized. Assuming that the phases of the functions $\phi_{k}(z)$, which are determined by the local properties of the random potential and by the time $t_{0}$, are random, uncorrelated functions for different momenta, the BEC density is given by

$$
n_{0}(z) \equiv\left\langle|\psi(z)|^{2}\right\rangle=2 \int_{0}^{\infty} \mathrm{d} k \mathcal{D}(k)\left\langle\left|\phi_{k}(z)\right|^{2}\right\rangle,
$$

where we have taken into account that $\mathcal{D}(k)=\mathcal{D}(-k)$ and $\left\langle\left|\phi_{k}(z)\right|^{2}\right\rangle=\left\langle\left|\phi_{-k}(z)\right|^{2}\right\rangle$.

We now briefly outline the properties of the functions $\phi_{k}(z)$ from the theory of localization of single particles. For a weak random potential, using the phase formalism [28] the state with momentum $k$ is written in the form:

$$
\phi_{k}(z)=r(z) \sin [\theta(z)] ; \partial_{z} \phi_{k}=k r(z) \cos [\theta(z)],
$$

and the Lyapunov exponent is obtained from the relation $\gamma(k)=-\lim _{|z| \rightarrow \infty}\langle\log [r(z)] /|z|\rangle$. If the disorder is sufficiently weak, then the phase is approximately $k z$ and solving the Schrödinger equation up to first order in $\left|\partial_{z} \theta(z) / k-1\right|$, one finds [28],

$$
\gamma(k) \simeq\left(\sqrt{2 \pi} / 8 \sigma_{\mathrm{R}}\right)\left(V_{\mathrm{R}} / E\right)^{2}\left(k \sigma_{\mathrm{R}}\right)^{2} \widehat{c}\left(2 k \sigma_{\mathrm{R}}\right),
$$

where $E=\hbar^{2} k^{2} / 2 m$. Such a perturbative (Born) approximation assumes the inequality

$$
V_{R} \sigma_{\mathrm{R}} \ll\left(\hbar^{2} k / m\right)\left(k \sigma_{\mathrm{R}}\right)^{1 / 2},
$$

or equivalently $\gamma(k) \ll k$. Typically, Eq. (11) means that the random potential does not comprise large or wide peaks.

Deviations from a pure exponential decay of $\phi_{k}$ are obtained using diagrammatic methods [29], and one has

$$
\begin{aligned}
\left\langle\left|\phi_{k}(z)\right|^{2}\right\rangle= & \frac{\pi^{2} \gamma(k)}{2} \int_{0}^{\infty} \mathrm{d} u u \sinh (\pi u) \times \\
& \left(\frac{1+u^{2}}{1+\cosh (\pi u)}\right)^{2} \exp \left\{-2\left(1+u^{2}\right) \gamma(k)|z|\right\},
\end{aligned}
$$

where $\gamma(k)$ is given by Eq. (10). Note that at large distances $(\gamma(k)|z| \gg 1)$, Eq. (12 reduces to $\left\langle\left|\phi_{k}(z)\right|^{2}\right\rangle \simeq$ $\left(\pi^{7 / 2} / 64 \sqrt{2 \gamma(k)}|z|^{3 / 2}\right) \exp \{-2 \gamma(k)|z|\}$. 
The localization effect is closely related to the properties of the correlation function of the disorder. For the 1D speckle potential the correlation function $\widehat{C}(k)$ has a high-momentum cut-off $2 / \sigma_{\mathrm{R}}$, and from Eqs. (4) and (10) we find

$\gamma(k)=\gamma_{0}(k)\left(1-k \sigma_{\mathrm{R}}\right) \Theta\left(1-k \sigma_{\mathrm{R}}\right) ; \gamma_{0}(k)=\frac{\pi m^{2} V_{\mathrm{R}}^{2} \sigma_{\mathrm{R}}}{2 \hbar^{4} k^{2}}$.

Thus, one has $\gamma(k)>0$ only for $k \sigma_{\mathrm{R}}<1$ so that there is a mobility edge at $1 / \sigma_{\mathrm{R}}$ in the Born approximation. Strictly speaking, on the basis of this approach one cannot say that the Lyapunov exponent is exactly zero for $k>1 / \sigma_{\mathrm{R}}$. However, direct numerical calculations of the Lyapunov exponent show that for $k>1 / \sigma_{\mathrm{R}}$ it is at least two orders of magnitude smaller than $\gamma_{0}\left(1 / \sigma_{\mathrm{R}}\right)$ representing a characteristic value of $\gamma(k)$ for $k$ approaching $1 / \sigma_{\mathrm{R}}$. For $\sigma_{\mathrm{R}} \gtrsim 1 \mu m$, achievable for speckle potentials [17] and for $V_{\mathrm{R}}$ satisfying Eq. (11) with $k \sim 1 / \sigma_{\mathrm{R}}$, the localization length at $k>1 / \sigma_{\mathrm{R}}$ exceeds $10 \mathrm{~cm}$ which is much larger than the system size in the studies of quantum gases. Therefore, $k=1 / \sigma_{\mathrm{R}}$ corresponds to an effective mobility edge in the present context. We stress that it is a general feature of optical speckle potentials, owing to the finite support of the Fourier transform of their correlation function.

We then use Eqs. (7), (12) and (13) for calculating the density profile of the localized BEC from Eq. (8). Since the highmomentum cut-off of $\mathcal{D}(k)$ is $1 / \xi_{\text {in }}$, and for the speckle potential the cut-off of $\gamma(k)$ is $1 / \sigma_{\mathrm{R}}$, the upper bound of integration in Eq. (8) is $k_{\mathrm{c}}=\min \left\{1 / \xi_{\text {in }}, 1 / \sigma_{\mathrm{R}}\right\}$. As the density profile $n_{0}(z)$ is a sum of functions $\left\langle\left|\phi_{k}(z)\right|^{2}\right\rangle$ which decay exponentially with a rate $2 \gamma(k)$, the long-tail behavior of $n_{0}(z)$ is mainly determined by the components with the smallest $\gamma(k)$, i.e. those with $k$ close to $k_{\mathrm{c}}$, and integrating in Eq. (8) we limit ourselves to leading order terms in Taylor series for $\mathcal{D}(k)$ and $\gamma(k)$ at $k$ close to $k_{\mathrm{c}}$.

For $\xi_{\text {in }}>\sigma_{\mathrm{R}}$, the high-momentum cut-off $k_{\mathrm{c}}$ in Eq. (8) is set by the momentum distribution $\mathcal{D}(k)$ and is equal to $1 / \xi_{\text {in }}$. In this case all functions $\left\langle\left|\phi_{k}(z)\right|^{2}\right\rangle$ have a finite Lyapunov exponent, $\gamma(k)>\gamma\left(1 / \xi_{\text {in }}\right)$, and the whole BEC wave function is exponentially localized. For the long-tail behavior of $n_{0}(z)$, from Eqs. (7), (8) and (12) we obtain:

$$
n_{0}(z) \propto|z|^{-7 / 2} \exp \left\{-2 \gamma\left(1 / \xi_{\text {in }}\right)|z|\right\} ; \quad \xi_{\text {in }}>\sigma_{\mathrm{R}} .
$$

Equation (14) assumes the inequality $\gamma\left(1 / \xi_{\text {in }}\right)|z| \gg 1$, or equivalently $\gamma_{0}\left(k_{\mathrm{c}}\right)\left(1-\sigma_{\mathrm{R}} / \xi_{\text {in }}\right)|z| \gg 1$.

For $\xi_{\text {in }}<\sigma_{\mathrm{R}}, k_{\mathrm{c}}$ is provided by the Lyapunov exponents of $\left\langle\left|\phi_{k}(z)\right|^{2}\right\rangle$ so that they do not have a finite lower bound. Then the localization of the BEC becomes algebraic and it is only partial. The part of the BEC wave function, corresponding to the waves with momenta in the range $1 / \sigma_{\mathrm{R}}<k<1 / \xi_{\text {in }}$, continues to expand. Under the condition $\gamma_{0}\left(k_{\mathrm{c}}\right)\left(1-\xi_{\text {in }}^{2} / \sigma_{\mathrm{R}}^{2}\right)|z| \gg 1$ for the asymptotic density distribution of localized particles, Eqs. (8) and (12) yield:

$$
n_{0}(z) \propto|z|^{-2} ; \quad \xi_{\text {in }}<\sigma_{\mathrm{R}} .
$$

Far tails of $n_{0}(z)$ will be always described by the asymptotic relations (14) or (15), unless $\xi_{\text {in }}=\sigma_{\mathrm{R}}$. In the special case of $\xi_{\text {in }}=\sigma_{\mathrm{R}}$, or for $\xi_{\text {in }}$ very close to $\sigma_{\mathrm{R}}$ and at distances where $\gamma_{0}\left(k_{\mathrm{c}}\right)\left|\left(1-\xi_{\text {in }}^{2} / \sigma_{\mathrm{R}}^{2}\right) z\right| \ll 1$, still assuming that $\gamma_{0}\left(k_{\mathrm{c}}\right)|z| \gg 1$ we find $n_{0}(z) \propto|z|^{-3}$.

Since the typical momentum of the expanding BEC is $1 / \xi_{\text {in }}$, according to Eq. (11), our approach is valid for $V_{\mathrm{R}} \ll$ $\mu\left(\xi_{\text {in }} / \sigma_{\mathrm{R}}\right)^{1 / 2}$. For a speckle potential, the typical momentum of the waves which become localized is $1 / \sigma_{\mathrm{R}}$ and for $\xi_{\text {in }}<\sigma_{\mathrm{R}}$ the restriction is stronger: $V_{R} \ll \mu\left(\xi_{\text {in }} / \sigma_{\mathrm{R}}\right)^{2}$. These conditions were not fulfilled, neither in the experiments of Refs. [15, 16, 17], nor in the numerics of Refs. [15, 23, 24].

We now present numerical results for the expansion of a 1D interacting BEC in a speckle potential, performed on the basis of Eq. (1). The BEC is initially at equilibrium in the combined random plus harmonic potential, and the expansion of the BEC is induced by switching off abruptly the confining potential at time $t=0$ as in Refs. [15, 16, 17, 20]. The differences from the model discussed above are that the random potential is already present for the initial stationary condensate and that the interactions are maintained during the whole expansion. This, however, does not significantly change the physical picture.

The properties of the initially trapped BEC have been discussed in Ref. 222] for an arbitrary ratio $\xi_{\text {in }} / \sigma_{\mathrm{R}}$. For $\xi_{\text {in }} \ll \sigma_{\mathrm{R}}$, the BEC follows the modulations of the random potential, while for $\xi_{\text {in }} \gtrsim \sigma_{\mathrm{R}}$ the effect of the random potential can be significantly smoothed. In both cases, the weak random potential only slightly modifies the density profile [22]. At the same time, the expansion of the BEC is strongly suppressed compared to the non-disordered case. This is seen from the time evolution of the rms size of the BEC, $\Delta z=$ $\sqrt{\left\langle z^{2}\right\rangle-\langle z\rangle^{2}}$, in the inset of Fig. 11. At large times, the BEC density reaches an almost stationary profile. The numerically obtained density profile in Fig. 11 shows an excellent agreement with a fit of $n_{0}(z)$ from Eqs. (7), (8) and (12), where a multiplying constant was the only fitting parameter. Note that

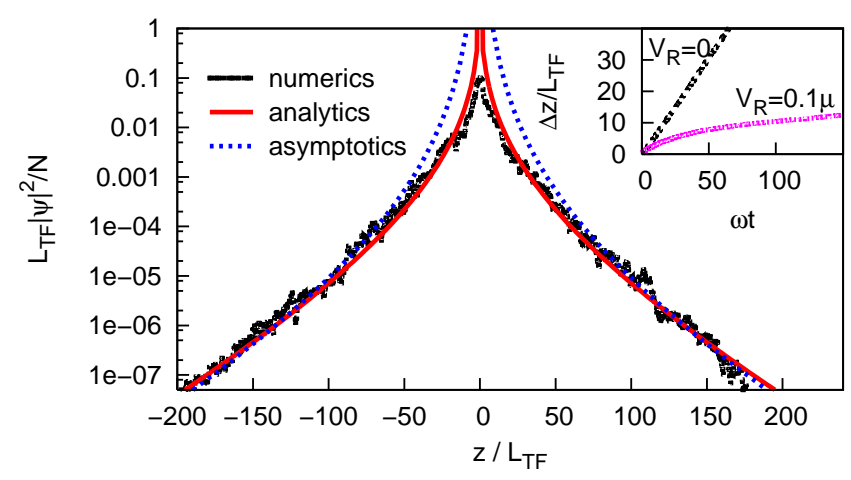

Figure 1: (color online) Density profile of the localized BEC in a speckle potential at $t=150 / \omega$. Shown are the numerical data (black points), the fit of the result from Eqs. (1), 82 and (12) [red solid line], and the fit of the asymptotic formula (14) [blue dotted line]. Inset: Time evolution of the rms size of the BEC. The parameters are $V_{\mathrm{R}}=0.1 \mu, \xi_{\text {in }}=0.01 L_{\mathrm{TF}}$, and $\sigma_{\mathrm{R}}=0.78 \xi_{\text {in }}$. 


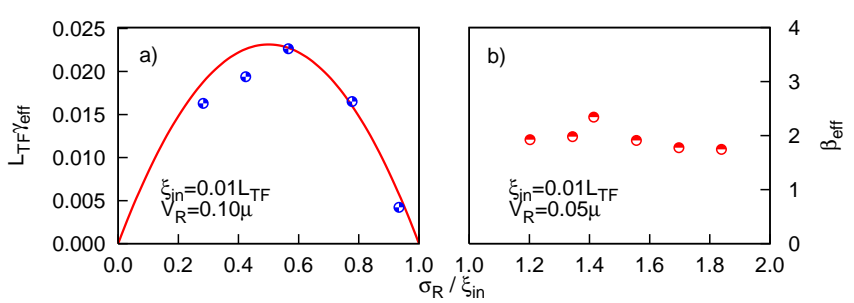

Figure 2: (color online) a) Lyapunov exponent $\gamma_{\text {eff }}$ in units of $1 / L_{\mathrm{TF}}$ for the localized BEC in a speckle potential, in the regime $\xi_{\text {in }}>$ $\sigma_{\mathrm{R}}$. The solid line is $\gamma\left(1 / \xi_{\text {in }}\right)$ from Eq. (13). b) Exponent of the power-law decay of the localized BEC in the regime $\xi_{\text {in }}<\sigma_{\mathrm{R}}$. The parameters are indicated in the figure.

Eq. (8) overestimates the density in the center of the localized BEC, where the contribution of waves with very small $k$ is important. This is because Eq. (13) overestimates $\gamma(k)$ in this momentum range, where the criterion (11) is not satisfied.

We have also studied the long-tail asymptotic behavior of the numerical data. For $\xi_{\text {in }}>\sigma_{\mathrm{R}}$, we have performed fits of $|z|^{-7 / 2} \mathrm{e}^{-2 \gamma_{\text {eff }}|z|}$ to the data. The obtained $\gamma_{\text {eff }}$ are in excellent agreement with $\gamma\left(1 / \xi_{\text {in }}\right)$ following from the prediction of Eq. (14), as shown in Fig. 2a. For $\xi_{\text {in }}<\sigma_{\mathrm{R}}$, we have fitted $|z|^{-\beta_{\text {eff }}}$ to the data. The results are plotted in Fig. 2 $2 \mathrm{~b}$ and show that the long-tail behavior of the BEC density is compatible with a power-law decay with $\beta_{\text {eff }} \simeq 2$, in agreement with the prediction of Eq. (15).

In summary, we have shown that in weak disorder the expansion of an initially confined interacting 1D BEC can exhibit Anderson localization. Importantly, the high-momentum cut-off of the Fourier transform of the correlation function for 1D speckle potentials can change localization from exponential to algebraic. Our results draw prospects for the observation of Anderson localization of matter waves in experiments similar to those of Refs. [15, 16, 17]. For $V_{\mathrm{R}}=0.2 \mu$, $\xi_{\text {in }}=3 \sigma_{\mathrm{R}} / 2$ and $\sigma_{\mathrm{R}}=0.27 \mu \mathrm{m}$, we find the localization length $L\left(1 / \xi_{\text {in }}\right) \simeq 460 \mu \mathrm{m}$. These parameters are in the range of accessibility of current experiments [17]. In addition, the localized density profile can be imaged directly, which allows one to distinguish between exponential and algebraic localization. Finally, we would like to raise an interesting problem for future studies. The expanding and then localized BEC is an excited Bose-condensed state as it has been made by switching off the confining trap. Therefore, the remaining small interaction between atoms should cause the depletion of the BEC and the relaxation to a new equilibrium state. The question is how the relaxation process occurs and to which extent it modifies the localized state.

We thank M. Lewenstein, S. Matveenko, P. Chavel, P. Leboeuf and N. Pavloff for useful discussions. This work was supported by the French DGA, IFRAF, Ministère de la Recherche (ACI Nanoscience 201), ANR (grants NTOR-442586, NT05-2-42103 and 05-Nano-008-02), and the Euro- pean Union (FINAQS consortium and grants IST-2001-38863 and MRTN-CT-2003-505032), the ESF program QUDEDIS, and the Dutch Foundation FOM. LPTMS is a mixed research unit 8626 of CNRS and University Paris-Sud.

* URL: http: //www.atomoptic.fr

[1] P. W. Anderson, Phys. Rev. 109, 1492 (1958).

[2] B. van Tiggelen, in Wave Diffusion in Complex Media, 1998 Les Houches Lectures, ed. J. P. Fouque (Kluwer, Dordrecht, 1999).

[3] A.F. Ioffe and A.R. Regel, Prog. Semicond. 4, 237 (1960).

[4] N.F. Mott and W.D. Towes, Adv. Phys. 10, 107 (1961).

[5] D.J. Thouless, Phys. Rev. Lett. 39, 1167 (1977).

[6] E. Abrahams et al., Phys. Rev. Lett. 42, 673 (1979).

[7] F.M. Izrailev and A.A. Krokhin, Phys. Rev. Lett. 82, 4062 (1999); F.M. Izrailev and N.M. Makarov, J. Phys. A: Math. Gen. 38, 10613 (2005).

[8] Y. Imry, Introduction to Mesoscopic Physics (Oxford, University Press, 2002).

[9] M.E. Gershenson et al, Phys. Rev. Lett. 79, 725 (1997).

[10] C.M. Aegerter et al, Europhys. Lett. 75, 562 (2006).

[11] T. Schwartz et al., Nature (London) 446, 52 (2007).

[12] For a review, see: D.M. Basko, I.L. Aleiner, and B.L. Altshuler, Ann. Phys. (N.Y.) 321, 1126 (2006).

[13] P. Horak et al., Phys. Rev. A 58, 3953 (1998); G. Grynberg et al., Europhys. Lett. 49, 424 (2000).

[14] J.E. Lye et al., Phys. Rev. Lett. 95, 070401 (2005).

[15] D. Clément et al., Phys. Rev. Lett. 95, 170409 (2005).

[16] C. Fort et al., Phys. Rev. Lett. 95, 170410 (2005).

[17] D. Clément et al., New J. Phys. 8, 165 (2006).

[18] L. Guidoni et al., Phys. Rev. Lett. 79, 3363 (1997).

[19] U. Gavish and Y. Castin, Phys. Rev. Lett. 95, 020401 (2005); T. Paul et al., Phys. Rev. A 72, 063621 (2005); R.C. Kuhn et al., Phys. Rev. Lett. 95, 250403 (2005).

[20] L. Sanchez-Palencia and L. Santos, Phys. Rev. A 72, 053607 (2005).

[21] B. Damski et al., Phys. Rev. Lett. 91, 080403 (2003); R. Roth and K. Burnett, Phys. Rev. A 68, 023604 (2003); A. Sanpera et al., Phys. Rev. Lett. 93, 040401 (2004); V. Ahufinger, et al., Phys. Rev. A 72, 063616 (2005); L. Fallani et al., Phys. Rev. Lett. 98, 130404 (2007); J. Wehr et al., Phys. Rev. B 74, 224448 (2006).

[22] L. Sanchez-Palencia, Phys. Rev. A 74, 053625 (2006); P. Lugan et al., Phys. Rev. Lett. 98, 170403 (2007).

[23] M. Modugno, Phys. Rev. A 73, 013606 (2006).

[24] E. Akkermans et al., cond-mat/0610579.

[25] Yu. Kagan et al., Phys. Rev. A 54, R1753 (1996): Y. Castin and R. Dum, Phys. Rev. Lett. 77, 5315 (1996).

[26] D.S. Petrov, G.V. Shlyapnikov, and J.T.M. Walraven, Phys. Rev. Lett. 85, 3745 (2000); D.S. Petrov, D.M. Gangardt, and G.V. Shlyapnikov, J. Phys. IV (France) 116, 3 (2004).

[27] J.W. Goodman, Statistical Properties of Laser Speckle Patterns, in Laser Speckle and Related Phenomena, J.-C. Dainty ed. (Springer-Verlag, Berlin, 1975).

[28] I.M. Lifshits, et al., Introduction to the Theory of Disordered Systems, (Wiley, New York, 1988).

[29] A.A. Gogolin et al., Sov. Phys. JETP 42, 168 (1976); A.A. Gogolin, ibid. 44, 1003 (1976). 